\title{
Sinderos
}

\section{La presencia antropológico-filosófica de Max Scheler en la obra de Paulo Freire: EI ser humano como ser de apertura ${ }^{1}$}

\author{
The anthropological-philosophical presence of Max Scheler in the \\ work of Paulo Freire: The human being as being of openness
}

\author{
Autor: \\ Diego Alejandro Muñoz Gaviria ${ }^{2}$ \\ Recibido: 16 de agosto de 2017 \\ Aprobado: 28 de mayo de 2018
}

\footnotetext{
1. Este escrito hace parte de la tesis doctoral titulada: "La concepción de lo humano y su educación - formación en la obra de Paulo Freire: Aportes para una antropología filosófica y pedagógica crítico-latinoamericana", adscrita al Doctorado en Filosofía de la Universidad Pontificia Bolivariana, Medellín.

2. Sociólogo, especialista en contextualización psicosocial del crimen, magíster en psicología y candidato a doctor en Filosofía. Profesor de la Facultad de Ciencias de la educación de la Universidad Católica de Oriente.
}

\section{Resumen}

Este texto pretende reconstruir, en las obras tempranas de Paulo Freire Educación como Práctica de la libertad (1969) y Pedagogía del Oprimido (1970), la presencia de la Antropología Filosófica Moderna de Max Scheler en su libro El Puesto del Hombre en El Cosmos (1928), lo que origina una Antropología Filosófica de la Apertura. La recepción que Freire hace de las tesis de Scheler se centra en la recuperación de la consideración del ser humano como ser excéntrico, es decir, llamado a romper consigo mismo para llegar a ser más. En Freire la excentricidad humana se concreta en tres aspectos o subcategorías antropológicas de la apertura: la diferencia ser humano - animal, la apertura humana y el diálogo humanizador. Queda como asunto para seguir investigando: la conexión, en Paulo Freire, de la excentricidad humana desde una antropología de la apertura con la relacionalidad y la liberación.

Palabras clave: Excentricidad, antropología filosófica de la apertura, diálogo humanizador, ser humano. 


\begin{abstract}
This text aims to reconstruct, in the early works of Paulo Freire Education as a Practice of Freedom (1969) and Pedagogy of the Oppressed (1970), the presence of Max Scheler's Modern Philosophical Anthropology in his book The Human Place in The Cosmos (1928), which originates a Philosophical Anthropology of the Openness. Freire's reception of Scheler's thesis focuses on the recovery of the consideration of the human being as an eccentric being, which means a being called to
\end{abstract}

break with himself to become more. In Freire, human eccentricity is specified in three aspects or anthropological subcategories of openness: the humananimal difference, human openness and humanizing dialogue. It remains as a matter to investigate: the connection, in Paulo Freire, of human eccentricity with relationship and liberation, based on an anthropology of openness.

Keywords: Eccentricity, philosophical anthropology of openness, humanizing dialogue, human being

\section{A modo de introducción}

Una vez más los hombres, desafiados por la dramaticidad de la hora actual, se proponen a sí mismos como problema. Descubren qué poco saben de sí, de su "puesto en el cosmos", y se preocupan por saber más. Por lo demás, en el reconocimiento de su poco saber de sí radica una de las razones de esa búsqueda. Instalándose en el trágico descubrimiento de su poco saber de sí, hacen de sí mismo un problema. Indagan. Responden y sus respuestas los conducen a nuevas preguntas Paulo Freire (1972)

La idea freiriana de reivindicar antropológicamente al humano como conjunto de relaciones (Muñoz, 2014) permite reconocer al ser humano, en la lectura críticohumanista, como ser de apertura, solo sería posible su relación con los otros, con lo otro y consigo mismo dado que es vida abierta a la experiencia y en constante excentricidad. Es esta tesis la que retoma el maestro Freire, principalmente en su texto Pedagogía del Oprimido (1972) ${ }^{3}$, de la denominada Antropología Filosófica Moderna o Escuela de Scheler, en cuanto se reconoce en la humana condición la centralidad de su excentricidad, su capacidad de romper consigo mismo, una idea expuesta en la obra de Scheler El puesto del hombre en el cosmos (1928) y desarrollada posteriormente por Plessner en su libro Los grados de lo orgánico en el ser humano (1928). Como lo recuerda el profesor Wulf:

En 1927 Max Scheler impartió en Darmstadt una conferencia titulada "el puesto singular del hombre en el cosmos”, que se publicó en 1928 con el título el puesto del hombre en el cosmos, y que es considerado el comienzo de la Antropología Filosófica. Scheler fallece ese mismo año y el proyecto anunciado de una obra

3. Pedagogía del Oprimido se publicó por primera vez en portugués en 1970, la versión en castellano aquí citada es la de 1972 de Siglo XXI editores. 
antropológica para 1929 queda interrumpido sin haber alcanzado una forma acabada. En cambio, el filósofo y biólogo Helmuth Plessner presentó en 1928 su obra antropológica más importante, Los grados de lo orgánico y el ser humano. A pesar de las grandes diferencias en el material y en la argumentación, los escritos de Scheler y el libro de Plessner se asemejan en el punto de partida, la construcción gradual de lo orgánico. De ello se aleja la investigación de Arnold Gehlen, El hombre, su naturaleza y su lugar en el mundo, que aparece en una versión expurgada de las resonancias nacionalistas y reelaborado en 1947, en cuyo centro figura el ser humano como ser de acción. (Wulf, 2008, pp. 52-53)

La tesis a sustentar en este escrito es: la presencia de Max Scheler en la obra de Paulo Freire se establece sobre el reconocimiento antropológico del ser humano como ser de apertura (Scheler, 1960, pp. 134,135). Para este diálogo académico es pertinente resaltar la base antropológica del pensamiento de estos autores, su antropología filosófica establecida en la premisa de la complejidad y problematicidad del ser humano, con ello sus diferencias con otras formas de vida. El ser humano es la única especie inconclusa que ha de tomar conciencia de su inacabamiento para hacer algo de sí misma. En el caso del filósofo alemán Max Scheler (1960), ${ }^{4}$ la pregunta por el ser humano se establece desde la consideración de su existencia como una existencia peculiar o diferente de lo animal, lo vegetal y lo inorgánico. La particularidad humana reside en su capacidad de romper con su propio centro, de ser capaz de verse a sí mismo como tema de reflexión y estudio. La excentricidad humana permite antropológicamente que el ser humano se reconozca como ser que puede romper con sus propios límites, un ser llamado a trascender los hechizos del entorno. Para el profesor Scheler:

[...] cabe decir que en ninguna época de la historia ha resultado el hombre tan problemático para sí mismo como en la actualidad. Por eso me he propuesto el ensayo de una nueva antropología filosófica sobre la más amplia base. En lo que sigue quisiera dilucidar tan sólo algunos puntos concernientes a la esencia del hombre, en su relación con el animal y con la planta, y al singular puesto metafísico del hombre - apuntando una pequeña parte de los resultados a que he llegado. (Scheler, 1960, p. 26)

Siguiendo a Scheler, Freire reconoce que el ser humano en los últimos tiempos se ha vuelto un "problema" para sí mismo y, en ese sentido, escribe: "El problema de su humanización [...] asume hoy el carácter de preocupación ineludible" (Freire, 1972, p. 31). Con ello Freire vincula sus ideas a esa tradición de pensamiento — antropología filosófica (alemana ${ }^{5}$ para la que el ser humano se ha vuelto problemático y que lleva a la cuestión por resolver acerca de su "puesto en el cosmos" (Scheler, 1960). Cuestión que surge en un momento en

4. El texto de Max Scheler "El puesto del hombre en el cosmos" fue editado por primera vez en 1928. La edición citada en este trabajo es de 1960, de la biblioteca filosófica de la editorial Losada.

5. Entre los precursores de la antropología filosófica están Max Scheler (1928), Helmuth Plessner (1928), Martin Heidegger (1927) y Arnold Gehlen (1940). 
el que, como lo plantea el mismo Scheler: "Estamos alrededor del año 10.000 de nuestra historia, en una época en la que el hombre se ha vuelto entera y totalmente 'problemático'; en una época en la que el hombre no sabe lo que es, pero que igualmente sabe que no lo sabe" (Scheler, 1960, p. 62). Freire parte igualmente de dicho reconocimiento de época: el ser humano no sabe lo que es, pero se ha vuelto igualmente consciente de que sabe que no lo sabe. La antropología filosófica se instaura — se despliega como saber- en este ámbito problemático (Freire, 1972). Por lo anterior, tanto Scheler como Freire reconocen en la condición humana una cuestión problemática que deberá ser abordada desde una lectura esperanzadora que proponga alternativas a la crisis antropológica contemporánea. Es de anotar que los dos autores, si bien comparten sus intuiciones antropológicas, generan en sus fundamentaciones y formas concretas de actuación diferencias políticas y pedagógicas.

En el caso de Scheler, su antropología filosófica centrada en la excentricidad humana asume políticamente una denuncia a la época que no consigue derivar en una práctica concreta de superación de la crisis. Podría decirse, siguiendo a Horkheimer $(2003)^{6}$, que en Scheler el idealismo y la metafísica no permiten derivar de su pensamiento una praxis concreta en contra de las condiciones materiales que imposibilitan la excentricidad humana7. Por ello, sus implicaciones pedagógicas, que pueden evidenciarse en las pedagogías de las ciencias del espíritu (Wulf, 2000, p. 44), sólo llegan hasta un llamado ético en el ser humano a asumirse responsable en su mundo de la vida, sin derivar de allí una radical transformación de la sociedad en su totalidad (Piñeres, 2017, p. 43) ${ }^{8}$.

\footnotetext{
6. Esta idea de Horkheimer se puede encontrar en los siguientes textos: Observaciones sobre ciencia y Crisis y teoría tradicional y teoría crítica. Estos textos han sido trabajados en este escrito en la versión incluida en el libro Teoría crítica de editorial Amorrortu (2003). Las críticas realizadas por Horkheimer a la moderna Antropología Filosófica de Scheler se pueden sintetizar en las siguientes ideas: el problema filosófico y político de creer que el ser humano es susceptible de ser reducido a una unidad estable en el devenir, lo cual implicaría una suerte de esencia de lo humano que se independiza de la historia; para la teoría crítica será fundamental establecer una lectura y propuesta antropológico-filosófica que dialoga con la historia, una suerte de antropología histórica que ubica dialécticamente las relaciones configuradas entre la historia y la acción humana concreta. En este sentido, es pertinente aclarar que, para el autor, toda lectura de la historia está cargada de intereses, éstos hacen parte de nuestros mundos de la vida y se manifiestan en nuestras miradas desde el presente al pasado, lo que hace de todo abordaje una lectura particular que no puede asumirse como absoluta o determinista. Por último, Horkheimer defiende unas categorías antropológicas que en su concreción histórica permitan defender la dignidad humana, protestando y transformando las condiciones de opresión que limitan o imposibilitan la realización de dicha dignidad.

7. Según Wulf, existen otras críticas a la Antropología Filosófica de Scheler, en primer lugar: "la descripción que hace Scheler de la capacidad del ser humano para objetualizar como rasgo distinto de la apertura al mundo no tiene suficiente alcance. Tanto la capacidad para la objetualización como para la observación imparcial tienen en su base elementos históricos y culturales, son resultado de un largo proceso civilizatorio que en nuestra cultura nos da hoy la posibilidad de percibir el mundo como lo hacemos (Wulf, 2008, p. 55). En segundo lugar: "tampoco ha podido superar la dicotomía, presente por lo menos desde Platón y Aristóteles, entre cuerpo y espíritu. Más bien prolonga la separación cartesiana de cuerpo y alma. Deudora de la intuición y ontología de esencias fenomenológicas y heredera de un concepto de persona y mundo tradicionales, la antropología de Scheler queda fuertemente atrapada en las corrientes filosóficas de su tiempo y por eso no ha trabajado con suficiente material empírico procedente de las ciencias naturales y humanas" (Wulf, 2008, pp. 55-56).

8. Para una lectura crítica a la Antropología Filosófica Moderna en clave de una antropología pedagógica negativa, ver la obra del profesor Juan David Piñeres Sus (2017). Lo humano como ideal regulativo. Imaginación antropológica: cultura, formación y antropología negativa (2017). Medellín: Universidad de Antioquia.
} 
Para Freire la antropología filosófica y su tesis sobre la excentricidad humana son fundamentales para pensar la humana condición, sin embargo, deberá complementarse con la pregunta en torno a las relaciones materiales de existencia (Muñoz, 2014). Para el pensador brasileño la complementariedad de la excentricidad, las relaciones materiales y la lucha por la libertad es indispensable en su Antropología Pedagógica Radical (Runge y Muñoz, 2016) ${ }^{9}$, es decir, aquella idea del ser humano centrada en la defensa política de las prácticas de libertad y pedagógica de la formación crítica y transformadora. La educación popular de Freire asume con mayor radicalidad o enraizamiento la praxis como fundamento para la comprensión y el cambio social. En cambio, la pedagogía de las ciencias del espíritu de Scheler sólo llega hasta la comprensión de la excentricidad humana.

Con el interés de sustentar estas ideas se proponen a continuación los siguientes ejes temáticos: en primer lugar, siguiendo a Max Scheler (1960), se propone una revisión de las tesis centrales de la antropología filosófica moderna en torno a la concepción del ser humano como ser excéntrico que dará piso a la configuración de una antropología filosófica de la apertura. En el segundo apartado del escrito se tematizan las principales recepciones y resignificaciones que Freire, como representante de la teoría crítica latinoamericana, realiza a las tesis de Scheler a partir de la identificación de tres "subcategorías antropológicas" o derivaciones de la antropología filosófica de la apertura: la diferenciación ser humano - animal, la apertura humana y el diálogo humanizador. Por último, se retoman algunas conclusiones que permiten rescatar la centralidad filosófica y pedagógica de la lectura de Freire a Scheler y algunas cuestiones abiertas que posibilitan futuras pesquisas sobre el tema.

\section{La antropología filosófica moderna como antropología de la apertura}

La Antropología Filosófica Moderna o Escuela de Scheler se consolida en Alemania en las primeras décadas del siglo XX. Sus ideas centrales se establecen con base en una relectura del neohumanismo alemán, principalmente de Hegel, y de la biología moderna posterior a Darwin (Wulf, 2008). El cruce de la filosofía y la biología posibilita en esta escuela una relectura de la pregunta por el ser humano en su doble implicación de ser excéntrico y orgánico.

El ser humano, en cambio, vive en una situación intermedia, en tanto Yo excéntrico. El centro de su posición le es accesible. Pero es excéntrico en tanto puede distanciarse de sí mismo. En ese sentido, puede encontrarse más acá y más allá del abismo abierto por la distancia. Está unido al cuerpo y al alma, pero, a su vez, se encuentra fuera de toda relación con el espacio y el tiempo. La vida del ser humano no puede romper su centralidad y, sin embargo, es excéntrico respecto a ella. (Wulf, 2008, p. 57)

\footnotetext{
9. Para Runge y Muñoz la Antropología Pedagógica Radical en Freire es "para mostrar la mencionada conexión, en este escrito, hemos tratado de restringirnos a las 'Primeras palabras' y al capítulo uno de Pedagogía del Oprimido. Nuestra tesis —si se quiere - es que en esas casi cincuenta páginas Paulo Freire no solo tiene como propósito esbozar su "programa pedagógico", su "pedagogía del oprimido", sino que sienta también allí las bases de una concepción antropológica que ha de ser considerada estrictamente en un sentido teórico-formativo, es decir, y como trataremos de mostrar, como una antropología pedagógica radical (Runge y Muñoz, 2016, p. 184).
} 
En los textos de 1928, El puesto del hombre en el cosmos (Scheler) y Los grados de lo orgánico en el ser humano (Plessner), se configuran las bases filosóficas y biológicas del intento de respuesta a la pregunta por el ser humano en clave antropológica moderna. Siendo el humano la única especie que tiene que desplegar sobre su propia existencia una suerte de trabajo vivo, su naturaleza sólo puede derivarse de su autoactividad, deja de ser una naturaleza preestablecida para llegar a ser una actividad de la apertura humana. Para estos autores, la principal diferencia del ser humano con los otros animales está en su capacidad de hacerse a sí mismo, con base en las condiciones biológicas e históricas que le son propias. El ser humano no nace al mundo con un proyecto plenamente trazado, al contrario, su natalidad establece la condición de un quehacer constante como humano. La antropología filosófica moderna tematiza esta autoactividad de la humanidad como excentricidad. En palabras de Scheler (1960, p. 64):

Si colocamos en el ápice del concepto de espíritu una función particular de conocimiento, una clase de saber, que sólo el espíritu puede dar, entonces la prioridad fundamental de un ser "espiritual" es su independencia, libertad o autonomía esencial —o la del centro de su existencia - frente a los lazos y a la presión de lo orgánico, de la "vida", de todo lo que pertenece a la "vida" y por ende también de la inteligencia impulsiva propia de ésta. Semejante ser espiritual ya no está vinculado a sus impulsos, ni al mundo circundante, sino que es "libre frente al mundo circundante.

Entre 1927 y 1928, Scheler propuso la excentricidad humana como la característica central de la humanidad. El ser humano es la única especie capaz de desprenderse de sí misma, de romper consigo misma. La excentricidad implica por ello la posibilidad de actuar sobre el propio ser, siendo posible que el ser humano se construya constantemente desde su excentricidad. Para Scheler sólo es posible comprender dicha excentricidad en tanto se mantenga actual la pregunta por el ser humano, el problema antropológico.

Los problemas que el hombre se plantea acerca de sí mismo han alcanzado en la actualidad el máximo punto que registra la historia por nosotros conocida. En el momento en que el hombre se ha confesado que tiene menos que nunca un conocimiento riguroso de lo que es, sin que le espante ninguna respuesta posible a esta cuestión, parece haberse alojado en él un nuevo denuedo de veracidad; el denuedo de plantearse este problema esencial de un modo nuevo, sin sujeción consciente — o sólo a medias o a cuartas partes consciente- a una tradición teológica, filosófica y científica, como era usual hasta aquí, el denuedo de desenvolver una nueva forma de conciencia y de la intuición de sí mismo, aprovechando a la vez ricos tesoros de saber especializado, que han labrado las distintas ciencias del hombre. (Scheler, 1960, p. 24)

Pese a esta problematización, el autor logra evidenciar que el puesto o el lugar del hombre en el cosmos cuenta con un aspecto central: Su excentricidad, o capacidad de romper con su propio centro. Con esta idea se deja claro que el ser humano es 
un ser que debido a su déficit instintivo está llamado a regularse espiritualmente. Como nidífugas o seres que rompen con su nido, los seres humanos desde la excentricidad requieren romper con las seguridades ofrecidas espiritualmente por una determinada civilización. El ser humano es un ser llamado a la excentricidad, a romper sus propios límites, lo cual conlleva efectos colaterales o perversos que se escapan al más elaborado estudio de prospectiva. Para Gehlen, esto permite entender que el ser humano es un ser en riesgo, destinado a buscar alternativas institucionales que permitan economizar su angustia y brindar algunas seguridades para la acción. Para Wulf, en Gehlen “en tanto ‘ser en riesgo', el ser humano necesita una seguridad cultural e institucional, así como auto-estabilización y disciplina” (2008, p. 62).

El devenir humano como excentricidad descansa esencialmente en una creciente metamorfosis de la tradición (Scheler, 1960, p. 50). Esta realidad convoca e incita a ir más allá de lo que se impone y está dada como la verdad; por esto es posible hoy hablar de diversidad o plurivocidad como manifestación de excentricidad (Wulf, 2008, p. 60). Es posible escuchar otras voces, otras formas o modos de ser. En el campo del conocimiento será posible transitar nuevos caminos de apertura y también de confluencia, este es el camino que se quiere transitar, de nuevas opciones, para construir puentes, para ver lo que a simple vista no se capta y con ello ampliar el horizonte de la pregunta por lo humano. La excentricidad posibilita, en una lectura antropológica, histórica y pedagógica, reconocer múltiples manifestaciones de lo humano y, por ello, diversas maneras de engendrarlo.

El ser humano es el ser que sabe decir no, el asceta de la vida, el eterno protestante contra toda mera realidad. En comparación también con el animal, es el eterno Fausto, la bestia cupidissima rerum novarum, nunca satisfecha de la realidad circundante, siempre ávida de romper los límites de su ser ahora, aquí y de este modo, de su "medio" y de su propia realidad actual (Scheler, 1960, p. 85). El ser humano tiene la posibilidad de no conformarse con lo que es dado por determinismos históricos y biológicos. Romper con sus límites es su formación constante como sujeto y como especie, de allí que su "naturaleza" no sea más que el cúmulo de autoactividad de los humanos humanizándose. Retomando a Plessner, Wulf expone lo siguiente:

En razón de la excentricidad, la relación del ser humano con el mundo no es inmediata; antes bien, se mediatiza en numerosos procesos, de tal modo que surge inmediatez mediada que caracteriza la relación del ser humano con el mundo. La excentricidad humana implica, por un lado, la imbricación con el mundo y, por otro, la capacidad para trazar fronteras y distanciarse. La mediación se produce en relación al mundo exterior a través de los sentidos, con la ayuda de sensaciones y emociones anímicas en el mundo interior y en relación a otros seres humanos en el mundo compartido. (Wulf, 2008, p. 60)

Esta excentricidad, entendida como posibilidad humana de romper con su propio centro, de convertir en cuestión su propia existencia, hace del ser humano una persona, un ser con posibilidades existenciales que puede hacer de sí un proceso. Para 
Scheler (1960, p. 63): “denominaremos persona, al centro activo en que el espíritu se manifiesta dentro de las esferas del ser finito, a rigurosa diferencia de todos los centros funcionales 'de vida', que, considerados por dentro, se llaman también centros anímicos".

La apertura al mundo es la concreción de dicha excentricidad, con ella, los seres humanos caminan por el mundo con el propósito fundamental de hacer algo consigo mismos. La pregunta pedagógica que complementa la idea filosófica de la excentricidad humana es la formabilidad. Así, un sujeto en constante devenir o metamorfosis es un ser basado existencialmente en la excentricidad y la formabilidad. Es de anotar que la capacidad de darse forma constantemente es la aspiración pedagógica de la denominada pedagogía de las ciencias del espíritu o pedagogía humanista alemana (Wulf, 2000). Los aportes antropológico-filosóficos de Scheler a la pedagogía se hacen evidentes en esta corriente alemana y en la constitución de la denominada Antropología Pedagógica Alemana (Runge, 2008).

En resumen, para Scheler y Plessner la categoría fundamental para tematizar la condición humana es la excentricidad. Esta categoría permite comprender la condición humana que permeará las bases humanistas de teorías críticas como la Escuela de Frankfurt y la Pedagogía Crítica latinoamericana. En este sentido, reivindicar al ser humano como ser excéntrico es abrir el camino para la defensa antropológicofilosófica de la emancipación humana, del proceso permanente de concienciaciónliberación. El ser que rompe con su entorno y con sí mismo es un ser crítico.

En la antropología de Freire, sus reflexiones sobre el ser humano en clave crítica, y por ende desde sus diálogos con autores como Marx y Fromm (Muñoz, 2014), reivindican al ser humano en condiciones concretas, no como especulación metafísica sino como sujeto concreto actuando en la historia. Como lo expone la profesora Stella Accorinti:

¿Cuál es nuestro puesto en el cosmos?, se preguntaba Paulo Freire parafraseando a Max Scheler, en el capítulo I de Pedagogía del Oprimido. Una primera aproximación a la respuesta está en la última parte del epígrafe de ese mismo capítulo: "Nadie libera a nadie, nadie se libera solo. Los seres humanos se liberan en comunión”. A esta liberación se llega por la praxis de la lucha, nos dice Freire. Esta lucha es un combate por el sentido, un combate que se libra entre todas y todos, porque el opresor no sólo está afuera, sino que, sobre todo, está adentro de cada uno. Esto constituye el miedo a la libertad, que Freire trabaja una y otra vez en sus obras, en su praxis. En este sentido, la libertad se conquista mediante el combate, la búsqueda y la propia praxis. El miedo a la libertad, que preocupó a Erich Fromm y a Paulo Freire, aún nos preocupa y nos ocupa, porque "decir que los hombres son personas, y como personas son libres y no hacer nada para lograr concretamente que esta afirmación sea objetiva, es una farsa”. En este sentido, es necesario recuperar la politicidad de la educación, como decía Freire, y esto tiene implicaciones éticas, gnoseológicas y epistemológicas, entre otras, ya que, como dice Peter Mc Laren, no sólo es imposible quitar la relación que la pedagogía tiene con la política, sino que hacerlo es, además, teóricamente deshonesto. (2006, p. 93) 
De allí que, para el caso de Freire, su antropología filosófica se fundamente en una antropología histórica, que contextualiza al ser humano preguntándose por sus realidades y existencias concretas y haciendo actual el postulado de la excentricidad. Al respecto escribe Freire:

De ahí la necesidad que se impone de superar la situación de opresión. Esto implica el reconocimiento crítico de la razón de esta situación, a fin de lograr, a través de una acción transformadora que incida sobre la realidad, la instauración de una situación diferente, que posibilite la búsqueda del ser más. (Freire, 1970, p. 38)

El reconocimiento del ser humano como ser de apertura, de su ser más, consigue una fundamentación en el diálogo antropológico-filosófico de Freire y Scheler a la luz de las siguientes inquietudes antropológicas: ¿cuál es la diferencia entre el ser humano y el animal? ¿Qué es la apertura humana? ¿Cómo se construye el diálogo en dicha apertura? A continuación, se tematizará cada una de estas preguntas como subcategorías antropológicas de la antropología de la apertura en Freire.

\section{Subcategorías antropológicas del ser humano como ser de apertura}

La recepción que Freire hace de las tesis de Scheler se encuentra explícitamente en lo que algunos biógrafos del pensador brasileño han denominado como el primer Freire (Gadotti, 1996; Rubens y Jardilino, 2009; Escobar, 2012; Villa y Muñoz, 2017). El primer momento de la obra de Freire (1962-1970) se ubica históricamente en el contexto del golpe militar que se produce en Brasil en 1964, cuando se detiene el proceso de educación de adultos y la cultura popular en general. Este periodo se caracteriza por la expulsión del autor de Brasil y por la obtención del asilo en Chile. En este país, el autor laboró en el Instituto de Capacitación e Investigación de la Reforma Agraria (ICIRA) y como docente de la Universidad Católica de Chile, al tiempo que cumplía labores de asesoría pedagógica en la Unesco (Villa y Muñoz, 2017, p. 278). En esta primera etapa del pensar de Freire se consolida su sistema teórico central, configurado en torno a tres ideas centrales: la pregunta antropológica por la realidad del ser humano latinoamericano, de allí su reconocimiento de los "oprimidos" como base antropológica para pensar en posteriores emancipaciones; la idea pedagógica crítica de entender y proponer acciones educativas tendientes a la liberación opuestas a la lógica bancaria-instrumental, y acciones formativas tendientes a la concienciación o emancipación (Escobar, 2012, pp. 16-31). Este primer Freire, o el autor de la Antropología Pedagógica Radical (Runge y Muñoz, 2016), establece tres aspectos centrales, aquí denominados subcategorías antropológicas, en su apropiación de la excentricidad de Max Scheler: la diferencia hombre - animal, la importancia de la apertura humana y la potencia del diálogo como praxis liberadora (Hernández, 2010). 


\section{La necesaria diferenciación ser humano - animal}

Tanto Scheler como Freire inician su reflexión antropológico-filosófica con la necesaria diferenciación del ser humano y el animal. Para estos autores, la principal diferencia del ser humano se establece sobre su condición de reconocerse como ser inacabado, aunque los animales y las plantas son a su vez seres inacabados, es sólo el ser humano quien puede tomar consciencia de ello. A diferencia de las plantas, tanto los animales como los seres humanos pueden romper con las condiciones de su entorno, pueden desligarse de su contexto, sin embargo, sólo los seres humanos pueden romper consigo mismos, pueden descentrase de su existencia, es decir, pueden ser excéntricos. Esta es la tesis central del diálogo Freire - Scheler. En palabras de Freire: "A través de su permanente quehacer transformador de la realidad objetiva, los hombres simultáneamente crean la historia y se hacen seres histórico-sociales." (Freire, 1972, p. 119). Para Scheler y Freire, en la parte biológica tanto el hombre como el animal traen interiorizado su comportamiento: ambos tienen una carga instintiva. Sin embargo, el animal no trasciende dicha carga, sólo la reproduce, en el caso del ser humano, de lo que se trata es de aprender y de construir una cultura que trasciende las regulaciones instintivas ${ }^{10}$. En este sentido, la condición humana parte de cierto déficit instintivo, que será compensado por la cultura.

Por esto mismo, el animal no puede comprometerse. Su condición de ahistórico no le permite asumir la vida y, dado que no la asume, no puede construirla. Si no la construye, tampoco puede transformar su contorno, no puede, tampoco, saberse destruido en vida, puesto que no consigue prolongar el soporte donde ella se da: en un mundo significativo y simbólico, el mundo compresivo de la cultura y de la historia. Esta es la razón por la cual el animal no animaliza su contorno para animalizarse, ni tampoco se desanimaliza. En el bosque, como un zoológico, continúa como un "ser cerrado en sí mismo", tan animal aquí como allá. (Freire, 1972, p. 115).

El ser humano se constituye en el mundo sobre la experiencia de la transformación y creación del mundo, el ser humano se consolida en el mundo con una reflexión, a decir de Freire: "Si la vida del animal se da en un soporte atemporal, plano, igual, la existencia de los hombres se da en un mundo que ellos recrean y transforman incesantemente" (1972, p. 115). El ser humano es un ser de la praxis en cuanto su quehacer está basado en la construcción de su propia existencia, no puede exonerarse de la responsabilidad de producir su propia historia, no puede dejarse llevar por el cauce del tiempo, pues él mismo es quien crea lo histórico, su ser y estar en el mundo es lo que permite la configuración de la historia, es más, sin seres humanos no habría cuestión histórica. Para Freire: "los hombres, contrariamente del animal, no solamente viven, sino que existen y su existencia es histórica” (Freire, 1972, p. 119).

10. En este sentido escribe Azevedo: “ao se perceber um ser -no-mundo e un ser-com-outros, o ser humano se diferencia dos outros animais, pois não apenas sabe do mundo, mas sabe o porquê de saber do mundo” (2008, p. 42). 
La existencia del hombre es histórica en la medida en que reflexiona sobre su realidad y a partir de esa reflexión toma decisiones sobre su vida y con base en ella puede proporcionar algún aporte para transformar el mundo (Muñoz, 2015).

La transformación de la realidad, que es la praxis humana le hace un ser en riesgo, un ser que no sabe con certeza las consecuencias de sus acciones, pero que no puede dejar de actuar en el mundo, su radical diferencia con los animales proviene de esta condición, de su ser histórico.

La diferencia entre los dos, entre el animal, de cuya actividad, por no constituir "actos límites", no resulta una producción más allá de sí, y los hombres que, a través de su acción sobre el mundo, crean el dominio de la cultura y de la historia, radica en que solo estos son seres de la praxis. (Freire, 1972, p. 118)

La combinación de elementos de organización y conformación social, y de cambio con cierta autonomía y libertad individual, ofrecen la posibilidad de innovación social, en la especie humana, dado que el ser humano se conduce por la razón proponiendo motivos a la voluntad y, por ende, gobernándose a sí mismo (Muñoz, 2014) lo anterior, constituye la diferencia del hombre "entre estar en” y "estar con”. El "estar en" implica un reconocimiento de la realidad contextual y la vinculación con el mundo. El "estar con” enfatiza en la posibilidad humana de establecer relaciones culturales, mediadoras con el mundo (Hernández, 2010, p. 23).

De este modo, empezaremos reafirmando el hecho de que los hombres son seres de la praxis. Son seres del quehacer, y por ello son diferentes de los animales, seres del mero hacer. Los animales no 'admiran' el mundo. Están inmersos en él. Por el contrario, los hombres como seres del quehacer 'emergen' del mundo y objetivándolo pueden conocerlo y transformarlo con su trabajo. (Freire, 1972, p. 157)

En conclusión, pensar antropológicamente al ser humano es reconocer su capacidad excéntrica de romper consigo mismo, una capacidad que le hace diferente a los animales, no mejor o peor, sólo diferente. Lo que permite esta diferencia es focalizar la atención antropológico-filosófica en la pregunta por la apertura humana: ¿en qué consiste su desprenderse de sí?

\section{La apertura humana}

En las siguientes líneas se propone una argumentación temática de la subcategoría la apertura humana, planteada por Hernández (2010), frente a la visión comunitarista de Freire del otro como ser de apertura (Freire, 1972).

Para Freire, el ser humano como ser de apertura es el reconocimiento de la capacidad excéntrica del ser humano de verse a sí mismo en sus circunstancias y relaciones, si el ser humano no se reconoce a sí mismo no se puede abrir. En términos de 
Plessner, sólo lo que se cierra puede tener apertura ${ }^{11}$, sólo lo orgánico en el ser humano permite que éste en su reconocimiento-cierre, pueda relacionarse-abrirse hacia los otros y lo otro.

Si en la vida del animal, el aquí no es más que un "hábitat" con el que entra en contacto, en la existencia de los hombres el aquí no es solamente un espacio físico, sino un espacio histórico. Rigurosamente, para el animal no hay un aquí, un ahora, un allí, un mañana, un ayer, dado que, careciendo de conciencia de sí, su vivir es una determinación total. Al animal no le es posible sobrepasar los límites impuesto por el aquí, por el ahora, por el allí (Freire, 1972, p. 116).

El ser humano en su condición de apertura es un ser en el mundo, lo que implica que el sujeto está activamente en él, su ser es un estar, por ende, el sentido del mundo dependerá del sentido que el mismo ser humano le otorgue; la permanencia del "ser en el mundo" es afrontar en comunión aquello que mantiene el vínculo social y la construcción colectiva de identidades y subjetividades (Muñoz, 2015). "El mundo humano, que es histórico, se hace, para el "ser cerrado en sí mismo” mero soporte. Su contorno no le es problemático, sino estimulante. Su vida no es un correr riegos, en tanto que no sabe que los corre" (Freire, 1972, p. 115).

El cerrarse es sinónimo de reconocerse como ser, sólo con este cierre la apertura es posible. El ser humano enfrenta desde esta lectura antropológica de la apertura el reto de encontrarse en el encuentro con los otros y lo otro, de hacerse a sí mismo sólo en la apertura a los otros y lo otro. El ser humano es así un ser que sólo se puede configurar a sí mismo en la relación, de allí nuevamente las ideas de Freire sobre la importancia de reconocer que el ser humano no se libera ni educa sólo, lo puede hacer en sus relaciones dialógicas. El ser humano desde el reconocimiento de sí y del otro, parte de la idea de Freire según la cual cuando el hombre se reconoce a sí mismo puede reconocer al otro.

Al objetivar su mundo, el alfabetizado se reencuentra con él, reencontrándose con los otros y en los otros, compañeros de su pequeño "círculo de cultura". Se encuentran y reencuentran todos en el mismo mundo común y, de la coincidencia de las intenciones que los objetivan, surgen la comunicación, el diálogo que critica y promueve a los participantes del círculo. (Freire, 1972, p. 7)

Freire reconoce en el "alfabetizado" un ser que es capaz de verse en sus relaciones, por ende, un ser capaz de valorar la presencia de los otros en él, se destaca la presencia de los otros porque de esa alteridad es como se logra vincular el otro y entrelazarse "para sí" (Muñoz, 2014).

11. En palabras de Wulf (2008, p. 56), "a diferencia de las cosas, los cuerpos vivos tienen una relación con su límite, que tiene una doble función: cierra y abre hacia el 'exterior'. El límite sólo es comprendido adecuadamente si se percibe bajo aspecto del 'exterior' y el 'interior'. Este doble aspecto conlleva la cuestión de la relación entre organismo y entorno. A diferencia de las cosas inertes, el límite vincula la planta, el animal y el ser humano con el mundo de lo vivo”. 
Cobra conciencia de la palabra como significación que se constituye en su intención significante, coincidente con intenciones de otros que significan el mismo mundo. Éste, el mundo, es el lugar de encuentro de cada uno consigo mismo y con los demás. (Freire, 1972, p. 7)

En resumen, la lectura antropológico-filosófica de Freire sobre la apertura humana es una contribución humanista para reivindicar en el ser humano sus múltiples maneras de devenir, sus formas diversas de relacionarse, sus responsabilidades con sus acciones y las implicaciones de sus configuraciones en los otros y las otras. Por todo lo anterior, se hace fundamental para la propuesta de una antropología de la apertura, contar con el componente dialógico. Con el diálogo el ser humano logra concretar sus relaciones sociales, políticas, culturales y educativas.

\section{El diálogo humanizador}

Para Freire la interacción dialógica posibilita concienciar, es decir, adentrarse en un proceso de toma de conciencia, donde el ser humano en sus situaciones concretas y en relación con los otros rescata su voz. En este sentido Freire entiende la concienciación como:

La crítica para nosotros implica que el hombre comprenda su posición dentro de su contexto. Implica su injerencia, su integración, la representación objetiva de la realidad. De ahí que la concienciación sea el desarrollo de esta toma de conciencia. No será, por eso mismo, resultado de las modificaciones económicas, por grandes e importantes que sean. La crítica, como la entendemos nosotros, ha de resultar del trabajo pedagógico crítico apoyado en condiciones históricas propicias. (Freire, 1971, pp. 54-55)

El diálogo es un encuentro de subjetividades semejantes y diferentes donde se da una lucha entre las distintas actitudes, donde se puede combatir la arrogancia, el orgullo y la intolerancia; además, en el diálogo hay conflicto porque hay un proceso histórico de reconocimiento de posturas y subjetividades. Sin embargo, el ser dialógico que se suscita en Freire es el que se inmiscuye en una relación horizontal no autoritaria, es un sujeto que trata de abolir las inconsistencias de la "cultura del silencio" emprende su viaje dialéctico y descubre la radical necesidad del otro para comprenderse. Según el autor: “¿Cómo puedo dialogar, si temo la superación y si sólo con pensar en ella sufro y desfallezco? La autosuficiencia es incompatible con el diálogo" (Freire, 1972, p. 104)

El diálogo se hace humanizador en tanto reivindica en el sí mismo la voz creadora y creativa, y reconoce en el otro una fuente inagotable de posibilidades, de allí que el diálogo sea fundamentalmente un tacto humanizador, un encuentro ético con 
los otros ${ }^{12}$. Dialogar es asignarle colectiva e intersubjetivamente sentido al mundo, siempre en el horizonte de la vital vinculación con los otros, sólo será posible llegar a ser sí mismo si se parte de la importancia del estar en común unión con los otros.

El diálogo es, por tanto, el camino indispensable — dice Jaspers— no solamente en las cuestiones vitales para nuestro orden político, sino para todo nuestro ser. El diálogo sólo tiene estímulo y significado en virtud de la creencia en el hombre y en sus posibilidades, la creencia de que solamente llego a ser yo mismo cuando los demás también lleguen a ser ellos mismos. (Freire, 1971, p. 104)

Una de las influencias centrales en esta antropología dialógica de Freire es Martín Buber y sus tesis sobre el yo y el tú. En esta lectura antropológico-filosófica, el diálogo es la posibilidad vinculante existente entre seres humanos que se ven irrevocablemente convocados a tratarse, a relacionarse. Para Azevedo:

Uma fonte de importante contribuição para que Freire construísse o sentido de diálogo em sua pedagogia foi o pensamento de Martín Buber. O pensamento buberiano reflete sobre a existência humana, essencialmente pela explicitação da relação dialógica entre EU e o TU, como forma de compreender o próprio sentido ontológico e essencial da convivência interpessoal entre os seres humanos e de sua relação com Deus. (Azevedo, 2008, p. 122)

\section{A modo de cierre}

La reflexión antropológico-filosófica del "ser humano como apertura" permite, en la reconstrucción de la obra de Freire y su lectura a Scheler, establecer una base antropológica que reivindica en la condición humana su llamado existencial a establecer vínculos y relaciones con los otros, las otras y con lo otro. En la existencia del ser humano, su apertura configura una mediación con el mundo, es decir, genera en él la necesidad de crearse en contacto - relación con los demás, porque sin el otro no hay una construcción de mi propio ser, de mi actitud, de mi lenguaje, de mi identidad (Muñoz, 2015).

Categorías como el ayer, el hoy y el mañana, no son secciones cerradas e intercomunicables en el tiempo, que quedan petrificadas y en las cuales los hombres encuentran enclaustrados. Si así fuere, desaparecería una condición fundamental de la historia, su continuidad. Las unidades epocales, por el contrario, están relacionadas las unas con las otras, en la dinámica de la continuidad histórica. (Freire, 1972, p. 119)

\footnotetext{
12. Para Azevedo: "a dialogicidade, enquanto concepção existencial dos seres humanos, é uma categoria determinante pra Freire na formulação de sua pedagogia e de seu humanismo. A filosofia existencialista é o grande aporte conceitual em que ele vai se apoiar teoricamente para desenvolver os princípios fundantes da educação dialógica, razão de ser da sua própria pedagogia libertadora. O reconhecimento dos seres humanos como seres abertos - ontologicamente inconclusos e vocacionados para o ser mais -, que não estão apenas no mundo, mas com o mundo, num permanente processo relacional, implica a firmação de que os homens e mulheres são existencialmente seres de comunicação" (2008, p.122)
} 
De otro lado, el efecto liberador del ser humano como apertura se encuentra en la posibilidad de ser éste un sujeto histórico, no sólo un reproductor de cultura, sino también un productor de cultura, es un heredero que reflexiona con rigor lo que se puede hacer desde lo que se está siendo (Muñoz, 2015). El llamado liberador de la apertura reconoce en los seres humanos seres que están en búsqueda de superar limitaciones y de sustentar su subjetividad como constructora de vida.

En la medida en que emerge del tiempo, liberándose de su unidimensionalidad, discerniéndola, sus relaciones con el mundo se impregnan de un sentido consecuente. En verdad, se afirma que la posición común del hombre en el mundo, visto no sólo como estando en él sino como él, no se agota en mera pasividad. Puede interferir, ya que no sólo se reduce a una de las dimensiones de las que participa — la natural y la cultural—, de la primera por su aspecto biológico, de la segunda por su poder creador. Su injerencia, salvo por accidente o distorsión, no le permite ser un simple espectador, a quien no le es lícito interferir en la realidad para modificarla. Heredando la experiencia adquirida, creando y recreando, integrándose a las condiciones de su contexto, respondiendo a sus desafíos, objetivándose a sí mismo, discerniendo, trascendiendo, se lanza el hombre a un dominio que le es exclusivo - el de la historia y de la cultura. (Freire, 1971, pp. 30-31)

Tanto para Freire como para Scheler, el ser humano ante la construcción del mundo se auto-realiza, porque genera una conciencia universal que le permite dar y recibir. En la medida en que el ser humano recibe, se abre y así descubre lo que el otro tiene para ofrecer, porque cada día que pasa la vida sorprende con experiencias que realizan y equilibran a la vez que sumergen en relaciones necesarias y viables, una particularidad existencial del ser humano que le hace diferente a otras especies.

Por lo tanto, mientras el animal es esencialmente un ser acomodado y ajustado, el hombre es un ser integrado. Su gran lucha viene siendo, a través del tiempo, la de superar los factores que lo hacen acomodado o ajustado. Es la lucha por su humanización amenazada constantemente por la opresión que lo ahoga, casi siempre practicada - y eso es lo más doloroso- en nombre de su propia liberación. (Freire, 1971, p. 32)

Queda por desarrollar ¿cómo desde la base antropológica del reconocimiento del ser humano en sus relaciones y aperturas se configura un sentido de libertad, una idea de ser humano como ser libre? 


\section{Referencias}

Accorinti, S. (2006). Práxis político-pedagógica de Paulo Freire. En M. Gadotti (ed.), Lecciones de Paulo Freire, cruzando fronteras: experiencias que se complementan (pp. 93-97). Buenos Aires: Consejo Latinoamericano de Ciencias Sociales

Azevedo (2008). Pedagogía Da humanização. A pedagogía humanista de Paulo Freire. São Paulo: Paulus editorial.

Escobar, M. (2012). Pedagogía Erótica. Paulo Freire y el EZLN. México: UNAM.

Freire, P. (1971). La educación como práctica de la libertad. México: Siglo XXI Editores.

Freire, P. (1972). Pedagogía del Oprimido. México: Siglo XXI Editores.

Gadotti, M. (1996). Paulo Freire Una Biobibliografía. Sâo Paulo: IPF.

Hernández, O. A. (2010). Hacia una antropología de la educación en América Latina desde la obra de Paulo Freire. Revista Magistro, 4(8), 19-32. Bogotá: Ed. Universidad Santo Tomás.

Horkheimer, M. (2003). Observaciones sobre la Antropología Filosófica. En Teoría crítica (pp. 50-75). Buenos Aires: Amorrortu Editores.

Muñoz, D. A. (2014). Antropología Filosófica Relacional en la obra de Paulo Freire: Diálogos con la Teoría Crítica de Karl Marx. Revista Perseitas, 2(2), pp. 186-203

Muñoz, D. A. (2015). Una aproximación filosófica y pedagógica a la obra de Paulo Freire: Hacia una antropología filosófico-pedagógica latinoamericana. En Modernidad y política. Sobre la pregunta antropológica. Medellín: Unaula.

Piñeres, J. (2017) Lo humano como ideal regulativo. Imaginación antropológica: cultura, formación y antropología negativa. Medellín: Universidad de Antioquia

Rubens, J. y Jardilino, L. (2009). Paulo Freire: Apuntes bio-bibliográficos. Bogotá: Editorial Magisterio.

Runge, A. (2008). Ensayos sobre pedagogía alemana. Bogotá: UPN.

Runge, A. y Muñoz, D. (2016). La Antropología Pedagógica Radical de Paulo Freire: Formabilidad, inacabamiento, formación y educación del ser humano. En Pedagogía crítica latinoamericana y género. Bogotá: Siglo del Hombre.

Scheler, M. (1960). El puesto del hombre en el cosmos. Buenos Aires: Losada.

Villa, E. y Muñoz, D. (2017, enero-julio). Paulo Freire en la educación popular latinoamericana: El porqué y el para qué de estarse formando como pueblo político. Revista Kavilando, 9(1), 276-286.

Wulf, C. (2000). Introducción a la ciencia de la educación. Entre teoría y práctica. Medellín: Universidad de Antioquia.

Wulf, C. (2008). Antropología, historia, cultura, filosofía. México: Anthropos. 